\title{
Solar System Wave Function and its Achievements
}

\author{
Abolfazl Soltani \\ Department of Physics, University of Birjand, Birjand, Iran
}

Email: soltani.a.phyics@gmail.com

\begin{abstract}
Pluto, Ceres and all planets of Solar system except Neptune, with a high approximation, follow a rule called Titius-Bode rule or Bode rule, which can by no means be considered as a stochastic event. This rule shows that the distance of the planets from the sun in Solar system is regulated. Here, I prove that the existence of a standing and cosine wave packet in solar system, with the wavelength $\lambda=0.6 A U$ ( $A U$ represents the distance of earth from the sun), is the reason for Bode rule. And moreover, I prove that this huge wave packet belongs to the sun. In this Article, based on the solar system wave function, we will enter into the atomic field and arrive to a new atomic model that helps us to describe many phenomena such as the normal Zeeman effect.
\end{abstract}

Keywords: Solar system, Titius-Bode rule, Quantum mechanics, Atomic model, Schroedinger equation, Thought experiment, Normal Zeeman effect

\section{Introduction}

The planets of solar system move around the sun in elliptical orbits such that the sun is in one of the focal points of these ellipses. These ellipses are very close to the circle, and in fact the orbits of the planets of solar system are concentric circles. Pluto, Ceres and all planets of Solar system except Neptune, with a high approximation follow a rule known as Bode rule or Titius-Bode rule. According to this rule, the distance of each planet from the sun is equal to $a=0.4 A U+0.3 A U \times 2^{n}$, where $0.4 A U$ is the distance of Mercury from the sun (or more precisely the length of the semi-major axis of Mercury's orbit) and $n=0,1,2,3, \ldots$. [1]. Table. 1 shows the high accuracy of the Bode rule. If this rule was only true for three or four planets, then we could call it a coincidence, but when it is true for seven planets, plus Ceres and Pluto, there is definitely a reason for it. It was historically based on this rule that Ceres was discovered in 1801 [1]. In this article, I will find the reason for the existence of the Bode rule. In fact, I will prove that the presence of a cosine and standing wave packet in solar system is the reason for existence of Bode rule; And moreover, I will prove that this wave packet belongs to the sun. Bode rule does not predict the distance of Neptune from the sun but, this article is able to give us the distance of Neptune. In this paper and in the "Elliptical orbits" section, we prove that each model for explain the formation of solar system without considering the solar system wave function is incomplete and wrong.

Next, using the model of solar system standing wave, we enter into the fundamental topics of quantum mechanics and arrive at an atomic model that has both the Schrodinger wave function and the Bohr atomic model inside. My atomic model explains why the Bohr atomic orbits are quantized (or In other words why the orbital angular momentum is quantized: $L=n \hbar$ ). Niels Bohr, neither in her famous article [2] nor in the years that followed, could not explain the reason for this 
event. The new atomic model predicts the existence of secondary lines in the hydrogen spectrum ${ }^{1}$. These are lines that neither the Bohr atomic model nor any other model has been able to explain [3][4][5][6]. Moreover, based on our new atomic model, we explain the reason of the normal Zeeman effect, etc. This article reveals what the nature of matter wave in quantum mechanics and what the nature of dark matter is. In this article, we prove that the potential function must be removed from the Schrodinger equation. Moreover, we prove that the results of a thought experiment are not reliable and cannot be used.

Based on this article, astronomy, astrophysics, cosmology, quantum mechanics, atomic and molecular physics and chemistry will enter into a new stage.

\begin{tabular}{|c|c|c|c|}
\hline Planet & $\begin{array}{c}\text { T-B rule distance } \\
\text { (AU) }\end{array}$ & $\begin{array}{c}\text { Semi-major } \\
\text { axis (AU) }\end{array}$ & $\begin{array}{c}\text { Deviation from } \\
\text { prediction }\end{array}$ \\
\hline Mercury & 0.4 & 0.39 & $-2.5 \%$ \\
\hline Venus & 0.7 & 0.72 & $+2.8 \%$ \\
\hline Earth & 1.0 & 1.00 & $0.00 \%$ \\
\hline Mars & 1.6 & 1.52 & $-4.77 \%$ \\
\hline Ceres & 2.8 & 2.77 & $-1.16 \%$ \\
\hline Jupiter & 5.2 & 5.20 & $+0.00 \%$ \\
\hline Saturn & 10.0 & 9.58 & $-4.45 \%$ \\
\hline Uranus & 19.6 & 19.20 & $-1.95 \%$ \\
\hline Pluto & 38.8 & 39.48 & $+1.05 \%$ \\
\hline
\end{tabular}

Table. 1. Planets distances from the sun and the prediction of Bode rule. Bode rule cannot predict the distance of Neptune from the sun.

\section{Wave Function and Bode Rule}

Consider a standing and cosine wave function with a wavelength $\lambda=0.6 \mathrm{AU}$ in solar system; if we assume that the first node of this wave is at a distance of $0.1 \mathrm{AU}$ from the sun the next nodes are at the distances of $0.4 A U, 0.7 A U, 1 A U, 1.3 A U, 1.6 A U, \ldots 2.8 A U, \ldots$ from the sun. Each node is $0.3 A U$ ahead of the previous node. If we consider the planets of solar system in the position of the nodes of this wave, in such a case, there is no planet on the first node ( $0.1 A U)$ and Mercury is on the second node, Venus is on the third node, earth is on the fourth node, Mars is on the sixth node, and the position of fifth node (1.3 $A U)$ is empty. The seventh, eighth, and ninth nodes are empty, and Ceres is on the tenth node. Jupiter is placed on the eighteenth node and Saturn is on the thirty-third node, and Uranus, Neptune, and Pluto are on the nodes farther from the sun. As you can see, a wave function, with the wavelength $\lambda=0.6 \mathrm{AU}$, easily predicts the position of the planets and it seems that a huge and standing wave plays a role in determining the position of the planets in solar system. Therefore, we can consider the reason for the Bode rule to be the existence

\footnotetext{
${ }^{1}$ Two series of lines appear in the spectrum of the hydrogen lamps. One series are the Ballmer, Lyman, Paschen, . . .. series. and the other lines called the secondary spectrum, which their number is very numerous and does not follow Rydberg equation: $\frac{1}{\lambda}=R\left(\frac{1}{n_{\text {low }}^{2}}-\frac{1}{n_{u p}^{2}}\right)$. Of course, this secondary spectrum is now known as the molecular spectrum, which this issue is based on Merton's article [3]. In this article, we show that this is wrong and only a part of the secondary spectrum lines is relevant to the hydrogen molecule.
} 
of a large cosine wave in solar system that oscillates along the axis perpendicular to the plane of solar system. I call this wave "Solar system wave function". In this article, I will obtain the equation of this wave function. But what does this wave belong to? I answer this question in this article. The presence of a huge cosine wave in solar system seems strange at first sight, but quantum mechanics eradicates our surprise. Based on quantum mechanics, a wave packet can be assigned to each object which called the " associated wave " of that object, and this associated wave is the solution of the Schroedinger equation. In this article, I prove that the above standing and cosine wave function (Solar system wave function) is in the form of a solution of the Schroedinger equation and therefore, based on quantum mechanics, this wave must belong to an object in Solar system; I demonstrate that this object is the sun $^{2}$.

\section{Wave Function of Solar System}

As mentioned, a cosine and standing wave function, with the wavelength $\lambda=0.6 A U\left(k=\frac{2 \pi}{\lambda}=\right.$ $\left.\frac{10 \pi}{3}\right)$, can predict the position of the planets in solar system. First I want to derive the phase constant $\left(\emptyset_{0}\right)$ of this wave function. Any wave in which the variables $x$ and $t$ are entered as a combination of $k x \pm w t$ is a traveling wave [7]. For example, $\sin \left(k x-w t+\emptyset_{0}\right)$ is a traveling wave. Thus, a standing wave is in the form of $\cos (w t) \cos \left(k x+\emptyset_{0}\right)$ or $\sin (w t) \cos \left(k x+\emptyset_{0}\right)$ or $\sin (w t) \sin \left(k x+\emptyset_{0}\right)$ or $\cos (w t) \sin \left(k x+\emptyset_{0}\right)$. As mentioned, a cosine standing wave can predict the positions of planets. Therefore, the form of the standing wave of solar system must be either $\sin (w t) \cos \left(k x+\emptyset_{0}\right)$ or $\cos (w t) \cos \left(k x+\emptyset_{0}\right)$ (In the following, we choose one of these two forms). For the nodes of these two standing waves we have $\cos \left(k x+\emptyset_{0}\right)=0$. As mentioned previously, Mercury is on the second node of Solar system wave function (the second node corresponds to the phase $\frac{3 \pi}{2}$ because $\operatorname{Cos} \frac{3 \pi}{2}=0$ ). We have:

$$
x_{\text {Mercury }}=0.4 A U \Rightarrow \psi\left(x_{\text {Mercury }}\right)=0 \Rightarrow \operatorname{Cos}\left(k x_{M}+\emptyset_{0}\right)=0 \Rightarrow k x_{M}+\emptyset_{0}=\frac{3 \pi}{2} \stackrel{k=\frac{10 \pi}{3}}{\Longrightarrow} \emptyset_{0}=\frac{\pi}{6}
$$

Having $k$ and $\emptyset_{0}$, we can easily find the position of the other planets using the equation $k x+\emptyset_{0}=\frac{\pi}{2}, \frac{3 \pi}{2}, \frac{5 \pi}{2}, \ldots ., \frac{(2 m-1) \pi}{2}$ (Where $\mathrm{m}$ is the node number). For example

or

$$
k x_{\text {Venus }}+\emptyset_{0}=\frac{5 \pi}{2} \Rightarrow \frac{10 \pi}{3} x_{\text {Venus }}+\frac{\pi}{6}=\frac{5 \pi}{2} \Rightarrow x_{\text {Venus }}=0.7 \mathrm{AU}
$$

$$
\begin{aligned}
& k x_{\text {Earth }}+\emptyset_{0}=\frac{7 \pi}{2} \Rightarrow \frac{10 \pi}{3} x_{\text {Earth }}+\frac{\pi}{6}=\frac{7 \pi}{2} \Rightarrow x_{\text {Earth }}=1 \mathrm{AU} \\
& k x_{\text {Mars }}+\emptyset_{0}=\frac{11 \pi}{2} \Rightarrow \frac{10 \pi}{3} x_{\text {Mars }}+\frac{\pi}{6}=\frac{11 \pi}{2} \Rightarrow x_{\text {Mars }}=1.6
\end{aligned}
$$

\footnotetext{
${ }^{2}$ I am aware that Quantum mechanics, the Schroedinger equation, and the de Broglie wavelength relation can only be used for subatomic scale and objects. But, in this article, I prove that quantum mechanics is also valid in astronomical scale and I will obtain the shape of Schroedinger equation and de Broglie wavelength relation in astronomical scale.
} 
92 The distances of the other planets can also be calculated in the same way, which is quite consistent

93 with experience. According to the above equation $\left(k x+\emptyset_{0}=\frac{(2 m-1) \pi}{2}\right)$, Neptune is on the ninety94 eighth node, which corresponds to the phase $\frac{195 \pi}{2}$. Contrary to the Bode rule, which is not able to 95 predict the distance of Neptune, my wave theory predicts the position of Neptune. Therefore, a 96 cosine and standing wave function with $\emptyset_{0}=\frac{\pi}{6}$ and $k=\frac{10 \pi}{3}$ can be attributed to solar system. But 97 what is the general equation of this wave function? As mentioned, The function of solar system 98 must contain a component with the equation $\cos \left(\frac{10 \pi}{3} x+\frac{\pi}{6}\right)$ and on the other hand, this wave must 99 be standing so that the position of the nodes (planets) does not change. Therefore, as mentioned, 100 the form of solar system wave function must be either $\cos (\delta w t) \cos \left(\frac{10 \pi}{3} x+\frac{\pi}{6}\right)$ or $101 \sin (\delta w t) \cos \left(\frac{10 \pi}{3} x+\frac{\pi}{6}\right)$. There is no difference between $\cos (\delta w t)$ and $\sin (\delta w t)$ Because we 102 know from trigonometric identities that: $\cos (\delta w t)=\sin \left(\delta w t+\frac{\pi}{2}\right)$. Therefore, we choose the 103 function $\cos (\delta w t) \cos \left(\frac{10 \pi}{3} x+\frac{\pi}{6}\right)$ and then we will show that our choice is correct $(\delta$ is a constant 104 number that we will derive its value). Since solar system has a certain size and is not infinitely 105 wide, its wave function must be localized (a wave packet). If we consider an expression in the 106 form $e^{-\gamma x^{2}}$ (which is a Gaussian function and plays the role of a wave envelope) in the final 107 function of solar system, in such a case, the final equation is a localized wave or a wave packet ${ }^{3}$, (the 108 value of $\gamma$, which is a positive number, will be obtained in the following). Thus, the primary form 109 of the wave function of solar system is as follows (equation 1) and the planets are on the nodes of 110 this wave function (Fig. 1):

$$
\begin{cases}\psi(x, t)=C \cos (\delta w t) \cos \left(\frac{10 \pi}{3} x+\frac{\pi}{6}\right) e^{-\gamma x^{2}} & x \geq 0 \\ \psi(x, t)=C \cos (\delta w t) \cos \left(\frac{10 \pi}{3} x-\frac{\pi}{6}\right) e^{-\gamma x^{2}} & x \leq 0\end{cases}
$$

112 In equation 1, $\gamma, \mathrm{C}$ and $\delta$ are constant values and we obtain their values in this article. This is an 113 empirical equation which we will obtain it from mathematic methods in the next section.

\footnotetext{
${ }^{3}$ Although a Gaussian wave packet is also infinitely wide, but it is very localized.
} 


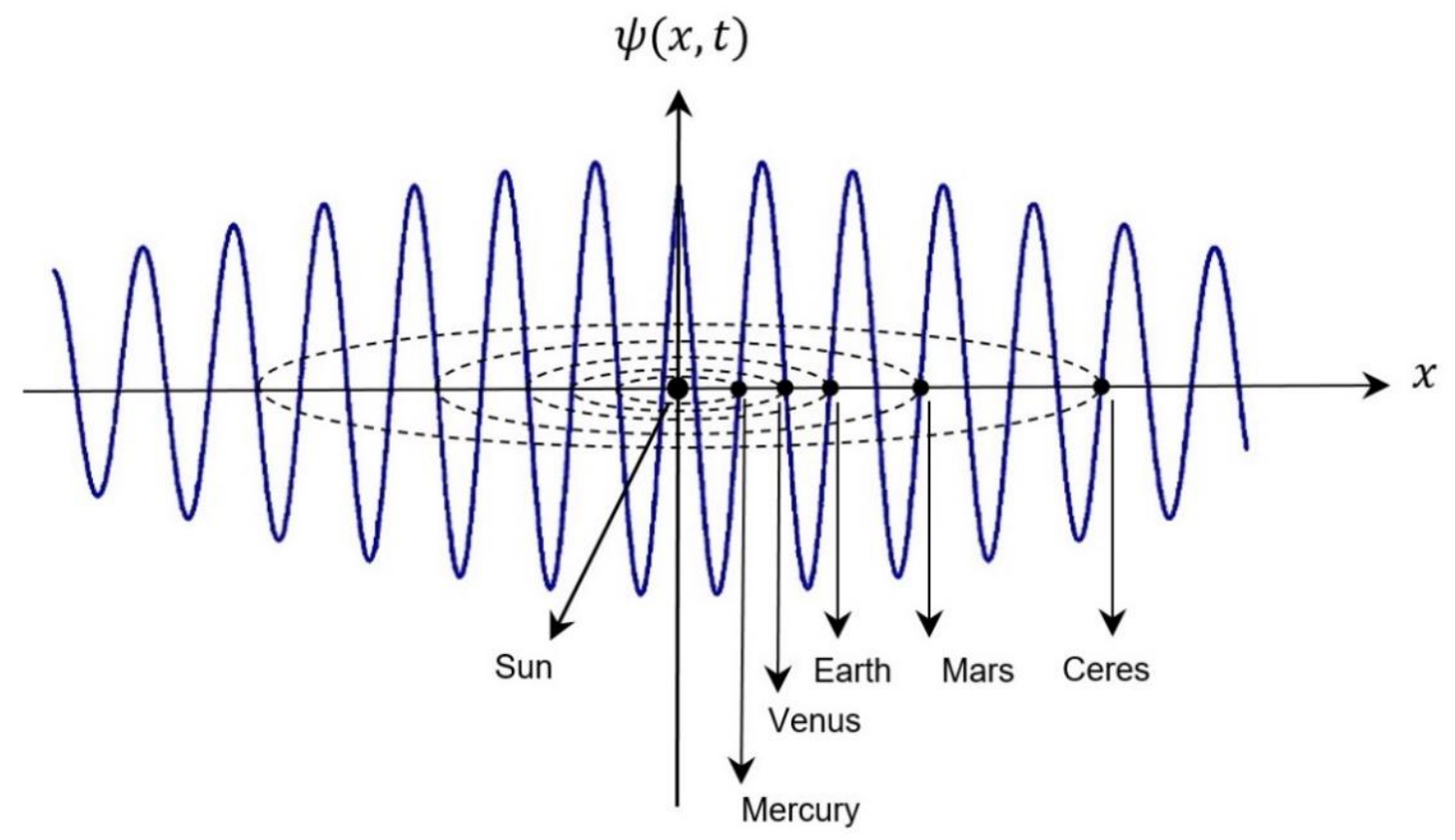

114

115

116

117

118

119

120

121

122

123

124

125

126

127

128

129

130

131

Fig. 1. Solar system standing wave packet with $\lambda=0.6 \boldsymbol{A U}$ and $\emptyset_{0}=\frac{\pi}{6}$. Diagram of $\psi(x, t)$ at the moment $\mathrm{t}=0$. The value of $\psi(0,0)$ equals $\sqrt{3} C / 2$. This diagram is drawn by a certain value of $C, \delta$ and $\gamma$ in equation 1 , which we will obtain their value in this article. As you can see, the planets are on the nodes of the wave function. Jupiter, Saturn, Uranus, Neptune, and Pluto are on the nodes farther from the sun.

In figure 1, the wave oscillates along the $\psi$ axis over time. But the nodes and the anti-nodes do not move relative to each other along the $\mathrm{x}$-axis. This does not mean that the wave packet is stationary in the space; it is just like passengers sitting on a train who do not move relative to each other but the train is moving relative to the rails. In the same way, solar system wave packet (equation 1) is a standing wave that rotates, along with solar system, around the center of the galaxy.

As you observed, function 1 could easily predicts the position of planets. In the continuation of the article, we will prove that this function is in the form of the Real part of a solution of the Schroedinger equation ${ }^{4}$ and that's why we can assign it to an object like the sun.

\section{Associated Wave Packet of Sun}

Consider a set of infinite numbers of flat matter waves $A e^{i\left(k x-w t+\emptyset_{0}\right)}$, which move in the positive direction of the $\mathrm{x}$-axis. As you know these waves are the solution of the Schrodinger equation for a free particle i.e. $i \hbar \frac{\partial}{\partial t} \psi(x, t)=-\frac{\hbar^{2}}{2 m} \frac{\partial^{2} \psi(x, t)}{\partial^{2} x}$. From these infinite numbers of waves we consider the waves which their angular frequency equals $w_{0}$ and the wave number of these waves is around

\footnotetext{
${ }^{4}$ As you know, the solutions of the Schroedinger equation have two parts: Real and Imaginary.
} 
132 the median of $k_{0}$ and between $k_{0}+\Delta k / 2$ and $k_{0}-\Delta k / 2$ and the amplitude changes of these waves is equal to $A(k)=\left(\frac{2 \alpha}{\pi}\right)^{1 / 4} e^{-\alpha\left(k-k_{0}\right)^{2}}$ (which is a Gaussian and symmetric function). In such a case, the resultant of these waves, using the superposition principle, is a wave packet with equation 2 [8][9][10].

$$
\psi_{\text {total }}(x, t)=\frac{1}{\sqrt{2 \pi}} \int_{-\infty}^{\infty} A(k) e^{i\left(k x-w_{0} t+\emptyset_{0}\right)} d k
$$

137 Where $\mathrm{k}$ means $k_{x}$. In equation $A(k)=\left(\frac{2 \alpha}{\pi}\right)^{1 / 4} e^{-\alpha\left(k-k_{0}\right)^{2}}, \alpha$ is a constant with a positive value and shows the width of the bell-shaped function $A(k) \cdot\left(\frac{2 \alpha}{\pi}\right)^{1 / 4}$ is a normalization coefficient which is obtained by normalize of $A(k)$. Since equation 2 is derived from the superposition principle, it is the solution of the Schrodinger equation [10].

To obtain $\psi_{\text {total }}(x, t)$ from equation 2 , we calculate the superposition of all of the waves in one moment, which we consider to be the origin of time $(t=0)$, and then we can obtain the net wave at any other time. We have:

$$
\psi(x, 0)=\frac{1}{\sqrt{2 \pi}} \int A(k) e^{i\left(k x+\emptyset_{0}\right)} d k
$$

The above equation is the momentary image of the net wave. Multiply equation 3 by $e^{i k_{0} x-i k_{0} x}$. We have:

$$
\psi(x, 0)=\frac{1}{\sqrt{2 \pi}} e^{i\left(k_{0} x+\emptyset_{0}\right)} \int A(k) e^{i\left(k-k_{0}\right) x} d k
$$

Considering $k^{\prime}=k-k_{0}$, we have:

$$
\psi(x, 0)=\left(\frac{\alpha}{2 \pi^{3}}\right)^{1 / 4} e^{i\left(k_{0} x+\emptyset_{0}\right)} \int e^{-\alpha k^{2}} e^{i k^{\prime} x} d k^{\prime}
$$

\footnotetext{
${ }^{5}$ In the Electromagnetic (EM) waves we cannot consider one $w_{0}$ for two or many waves in which their $k$ is different from each other, because for all of the EM waves we have: $w=c k$ where $c$ is the velocity of light. But for matter waves the issue is different. In the matter waves we have $w=\frac{\hbar k^{2}}{2 m}[10]$. As you can see $w$ is the function of $k$ and $m$. Therefore, it is possible to choose one value of $w_{0}$ for the waves in which their $k$ is different from each other. But In Quantum mechanics books, $w$ has been expanded about $k_{0}$ :

$$
w(k)=w\left(k_{0}\right)+\left(k-k_{0}\right)\left(\frac{d w}{d k}\right)_{k_{0}}+\frac{1}{2}\left(k-k_{0}\right)^{2}\left(\frac{d^{2} w}{d k^{2}}\right)_{k_{0}}+\ldots
$$

And physicists put this equation in equation 2 instead $w_{0}$. the expansion of $w$ about $k_{0}$ is due to the Heisenberg uncertainty principle. But in this article we will show that this is wrong. Let's talk about this issue in the section "Uncertainty principle and Single Frequency Wave packet" (section 8). The reader can read the section 8 before reading this section.
} 
150 Using the variable transformation $k^{\prime}-\frac{i x}{2 \alpha}=q$ [8][9] and the Gaussian integral $151 \int_{-\infty}^{\infty} d q e^{-\alpha q^{2}}=\sqrt{\frac{\pi}{\alpha}}$, equation 5 can be calculated. After replacement and simplification, we reach 152 the following final solution [8][9]:

$$
\psi(x, 0)=\left(\frac{\alpha}{2 \pi^{3}}\right)^{1 / 4} \sqrt{\frac{\pi}{\alpha}} e^{i\left(k_{0} x+\emptyset_{0}\right)} e^{-\frac{x^{2}}{4 \alpha}}=\left(\frac{1}{2 \pi \alpha}\right)^{1 / 4} e^{i\left(k_{0} x+\emptyset_{0}\right)} e^{-\frac{x^{2}}{4 \alpha}}
$$

154 Lets check the normalization

$$
\int_{-\infty}^{\infty}|\psi(x)|^{2} d x=\sqrt{\frac{1}{2 \pi \alpha}} \int_{-\infty}^{\infty} e^{-\frac{x^{2}}{2 \alpha}} d x=\sqrt{\frac{1}{2 \pi \alpha}} \sqrt{2 \alpha \pi}=1
$$

Given a normalized $A(k)$, we get the normalized $\psi(x)$.

Now, how is the time variation of equation 6? Let's go back to equation 2 :

$$
\psi(x, t)=\frac{1}{\sqrt{2 \pi}} \int A(k) e^{i\left(k x-w_{0} t+\emptyset_{0}\right)} d k=\left(\frac{\alpha}{2 \pi^{3}}\right)^{1 / 4} \int e^{-\alpha\left(k-k_{0}\right)^{2}} e^{i\left(k x-w_{0} t+\emptyset_{0}\right)} d k
$$

We put $e^{i k_{0} x-i k_{0} x}$ in the equation:

$$
\psi(x, t)=\left(\frac{\alpha}{2 \pi^{3}}\right)^{1 / 4} e^{i\left(k_{0} x+\emptyset_{0}\right)-i w_{0} t} \int e^{-\alpha k^{2}} e^{i k x} d k^{\prime}
$$

This integral is similar to integral 6, which led to $\psi(x, 0)$ (Equation 6). Therefore, we have:

$$
\begin{gathered}
\psi(x, t)=\psi_{1}(x, t)=\left(\frac{1}{2 \pi \alpha}\right)^{1 / 4} e^{i\left(k_{0} x-w_{0} t+\emptyset_{0}\right)} e^{-\frac{x^{2}}{4 \alpha}} \\
e^{i \theta}=\cos \theta+i \sin \theta \Rightarrow \operatorname{Re} \psi_{1}(x, t)=\left(\frac{1}{2 \pi \alpha}\right)^{1 / 4} \cos \left(k_{0} x-w_{0} t+\emptyset_{0}\right) e^{-\frac{x^{2}}{4 \alpha}}
\end{gathered}
$$

Due to the presence of the factor $k_{0} x-w_{0} t$, equations 8 and 9 represent a traveling wave packet that propagates in the positive direction of the $x$-axis [7]. This means that the location of the nodes is not known. Due to the absence of $t$ in $e^{-\frac{x^{2}}{4 \alpha}}$ in equations 8 and 9 , the wave packets in these equations does not spread.

Similarly, we use the recent trend to obtain the superposition of flat waves traveling in the negative direction of the $x$-axis, i.e. $A e^{i\left(k x+w_{0} t+\emptyset_{0}\right)}$. If we do this, we get to equation 9:

171

$$
\psi_{2}(x, t)=\left(\frac{1}{2 \pi \alpha}\right)^{1 / 4} e^{i\left(k_{0} x+w_{0} t+\emptyset_{0}\right)} e^{-\frac{x^{2}}{4 \alpha}}
$$

$$
\operatorname{Re} \psi_{2}(x, t)=\left(\frac{1}{2 \pi \alpha}\right)^{1 / 4} \cos \left(k_{0} x+w_{0} t+\emptyset_{0}\right) e^{-\frac{x^{2}}{4 \alpha}}
$$


172 This equation shows a traveling wave packet that propagates in the negative direction of 173 the $x$-axis.

174 Now we sum up the two equations 10 and 8 together to get the final wave.

$$
\operatorname{Re} \psi_{\text {total }}(x, t)=\operatorname{Re} \psi_{1}+\operatorname{Re} \psi_{2}
$$

Thus:

$$
\operatorname{Re} \psi_{\text {total }}(x, t)=\left(\frac{1}{2 \pi \alpha}\right)^{1 / 4} e^{-\frac{x^{2}}{4 \alpha}}\left[\cos \left(k_{0} x-w_{0} t+\emptyset_{0}\right)+\cos \left(k_{0} x+w_{0} t+\emptyset_{0}\right)\right]
$$

Using $\cos \alpha+\cos \beta=2 \cos \frac{1}{2}(\alpha+\beta) \cos \frac{1}{2}(\alpha-\beta)$ and $\cos (\theta)=\cos (-\theta)$ we obtain the equation of a standing wave packet.

$$
\left\{\begin{array}{l}
\alpha=k_{0} x-w_{0} t+\emptyset_{0} \\
\beta=k_{0} x+w_{0} t+\emptyset_{0}
\end{array} \Rightarrow \operatorname{Re} \psi_{\text {total }}(x, t)=2\left(\frac{1}{2 \pi \alpha}\right)^{1 / 4} \cos \left(k_{0} x+\emptyset_{0}\right) \cos \left(w_{0} t\right) e^{-\frac{x^{2}}{4 \alpha}}\right.
$$

There is not the structure of $k x \pm w t$ in equation 12 so the $\psi_{\text {total }}$ is a standing wave. As you observe, equation 12, which is the real part of a solution of the Schroedinger equation, is exactly the same as equation 1 for $x \geq 0$, which is solar system wave function. It means that equation 1 is the real part of a solution of the Schroedinger equation. It means that the Schroedinger equation and quantum mechanics are valid in astronomical scale. This is a great achievement in physics. by comparing equation 12 and equation 1 , we have

$$
\delta=1, \gamma=\frac{1}{4 \alpha} \quad \text { and } \quad C=2\left(\frac{1}{2 \pi \alpha}\right)^{1 / 4}
$$

If we put these values in equation 1, then we get the final equation of solar system wave function for $x \geq 0$ :

$$
\operatorname{Re} \psi_{t}(x, t)=2\left(\frac{1}{2 \pi \alpha}\right)^{1 / 4} \cos \left(w_{0} t\right) \cos \left(\frac{10 \pi}{3} x+\frac{\pi}{6}\right) e^{-\frac{x^{2}}{4 \alpha}} \quad x \geq 0
$$

Equation 13 is obtained by calculating the superposition of a set of infinite number of waves $A e^{i\left(k x-w_{0} t+\emptyset_{0}\right)}$ and $A e^{i\left(k x+w_{0} t+\emptyset_{0}\right)}$ that move in opposite directions to each other (pay attention to the + sign behind $\emptyset_{0}$ ). Now if we sum a set of infinite number of flat wave functions with the equations $A e^{i\left(k x-w_{0} t-\emptyset_{0}\right)}$ and $A e^{i\left(k x+w_{0} t-\emptyset_{0}\right)}$ (pay attention to the - sign behind $\left.\emptyset_{0}\right)$ together, by following the path we have taken from equation 2 to equation 13, we reach the following relation;

$$
\operatorname{Re} \psi_{t}(x, t)=2\left(\frac{1}{2 \pi \alpha}\right)^{1 / 4} \cos \left(w_{0} t\right) \cos \left(\frac{10 \pi}{3} x-\frac{\pi}{6}\right) e^{-\frac{x^{2}}{4 \alpha}}
$$

Which is the same as equation 1 for $x \leq 0$. Therefore, the final form of solar system wave function (equation 1) is as follows: 


$$
\begin{cases}\operatorname{Re} \psi(x, t)=2\left(\frac{1}{2 \pi \alpha}\right)^{1 / 4} \cos \left(w_{0} t\right) \cos \left(\frac{10 \pi}{3} x+\frac{\pi}{6}\right) e^{-\frac{x^{2}}{4 \alpha}} & x \geq 0 \\ \operatorname{Re} \psi(x, t)=2\left(\frac{1}{2 \pi \alpha}\right)^{1 / 4} \cos \left(w_{0} t\right) \cos \left(\frac{10 \pi}{3} x-\frac{\pi}{6}\right) e^{-\frac{x^{2}}{4 \alpha}} & x \leq 0\end{cases}
$$

In this equation, the larger the $\alpha$ is, the more the width of wave packet, along the $\mathrm{x}$-axis. We drew Fig. 1 by $\alpha=10$.

Here we demonstrated that solar system wave function (equation 1) is the real part of a solution of the Schroedinger equation. So, based on quantum mechanics, we can assign it to an object in Solar system. The closest star to solar system is at a distance of 4.8 light-years, which is so far. And the biggest and heavyset object in solar system is sun. Therefore, the wave function of solar system can only belong to the sun. In the " Elliptical orbits " section, we will discuss more about the formation of the solar system wave packet.

In this article, we proved that the Schroedinger equation is valid in astronomical scale; on the other hand, as you know, the Schroedinger relation is based on de Broglie equation $\left(\lambda=\frac{h}{m v}\right)$. Therefore, the de Broglie equation is valid in astronomical scale ${ }^{6}$. But, according to the very large mass of sun, using the de Broglie relation the wavelength $0.6 \mathrm{AU}$ will not obtain. So, instead of Planck constant we must choose another value for celestial objects, which is larger than $h$. We call this new value the Planck constant in Astronomy $\left(h_{\text {Astronomy }}\right.$ ) abbreviated as $h_{A}$ and we have: $\lambda_{A}=\frac{h_{A}}{P}$. In such a case, the Schrodinger equation for a free particle in the astronomical scale can be written as follows:

$$
i \hbar_{A} \frac{\partial}{\partial t} \psi(x, t)=-\frac{\hbar_{A}^{2}}{2 m} \frac{\partial^{2} \psi(x, t)}{\partial x^{2}}
$$

If we follow the path of proving the Schroedinger equation [11] and put the value $\hbar_{A}$ instead of $\hbar$, we reach equation 15. The Davisson-Germer experiment [12] is considered as the confirmation of existence of the de Broglie wave at the atomic scale, and the regularity of the distances of the planets from sun (Titius-Bode rule) is the confirmation of the existence of the de Broglie wave in astronomical scale. Moreover, for the celestial wave packet we will have $w=\frac{\hbar_{A} k^{2}}{2 m}$.

De Broglie considered the wave nature of light for subatomic particles, and here we attributed the wave nature to celestial objects. Neither of these two actions is strange. Rather, they are truths that we must become accustomed to.

\footnotetext{
${ }^{6}$ The Davisson-Germer experiment [12] is the confirmation of the existence of the de Broglie wave in subatomic scale and the regularity of the distances of the planets from sun (Titius-Bode rule) is the confirmation of the existence of the de Broglie wave in astronomical scale.
} 


\section{What is Waving?}

As you know, since the solution of the Schroedinger equation is a complex function; It is meaningless to talk about what is waving [13][14]. But in this section we will show that the real part of the solution of the Schroedinger equation is the oscillation of Dark matter.

In this article, we showed that a standing wave oscillation had been effective in the formation of orbits of solar system planets and created the Bode rule. On the other hand, we know that the Dark matter interacts with the matters and affects them. Therefore, it can be concluded that the standing wave oscillation in Fig. 1, with the equation 1, is the oscillation of Dark matter (Fig. 2). On the other hand, the equation 1 is the real part of a solution of the Schroedinger equation. Therefore, the real part of the solution of the Schroedinger equation is the oscillation of Dark matter. Of course, the Spacetime also interacts with matter and affects it. But we cannot consider the oscillation of Spacetime as the wave of the solar system. Because in such a case the curvature of Spacetime lines in figure 2 causes the planets to move along the r-axis.

As you will see in the next section, the oscillation of dark matter collected the gas and dusts of Protoplanetary disk, in certain orbits around the newborn sun and then gradually due to collision and gravitational attraction between the dusts and gas, the Protoplanets and then the planets in the orbits were formed.

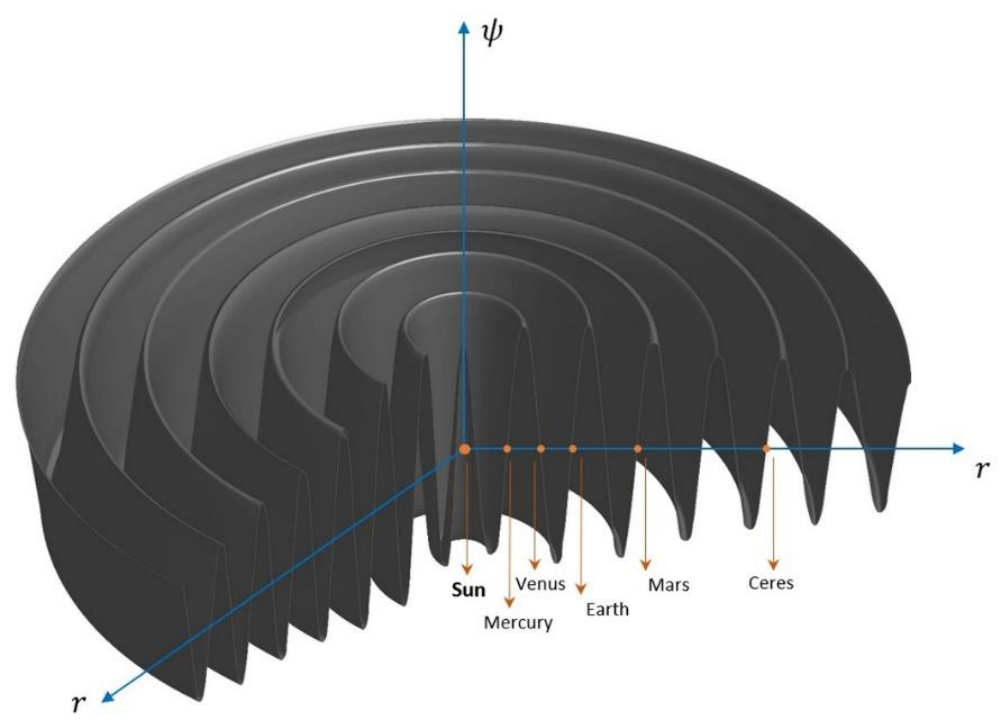

Fig. 2. The oscillation of Dark matter in the solar system. Here The wave function in Figure 1 is shown in three dimensions. Obviously, this wave function continues indefinitely, based on equation 1 (or equation 14). Because of existence the exponential factor in equation 1, the amplitude of the wave gradually decreases while we move away from the $\psi$-axis. Fig. 2 is drawn in cylindrical coordinates using equation 14.

\section{Elliptical orbits}

As you can see in Fig. 1 and Fig. 2, because of symmetry of equation 1, the orbits, created by the solar system wave function, are circle. But the real orbits of the planets are elliptic. The reason for 
this is the existence of the inverse_square gravitational force of the sun ${ }^{7}$. As you know, the sun formed earlier than the planets [15][16][17]. Simultaneously with the formation of the sun, about 4.6 billion years ago [16], its wave function was also formed ${ }^{8}$, and the oscillation of this wave function arranged and collected the dust particles and gas of the Protoplanetary disk in regular orbits (Bode orbit). Just like the standing wave patterns on the kettledrum head (Fig. 3) As you can observe in Fig.3, by a mechanical oscillator at the upper left of the photograph, the powder collects at the nodes [18]. Over time, and because of oscillation of dark matter, in some nodes of the Solar system wave function the dust grains agglomerated and then compressed due to collisions with each other and formed larger grains, protoplanets and finally planets ${ }^{9}$. At the same time, the inverse_square force of the sun was at work, and changed the circular orbits to elliptical orbits.

If solar system wave function did not exist; The planets had been orbited around the sun in elliptical orbits but the distance of planets from the sun was random and irregular. Without solar system wave packet, it is not possible to reach to the Titius-Bode rule from the molecular nebula and the protoplanetary disk. Thus, the existence of a standing wave in the solar system is undeniable. But, we do not know if solar system wave packet is a frozen standing wave or oscillating.

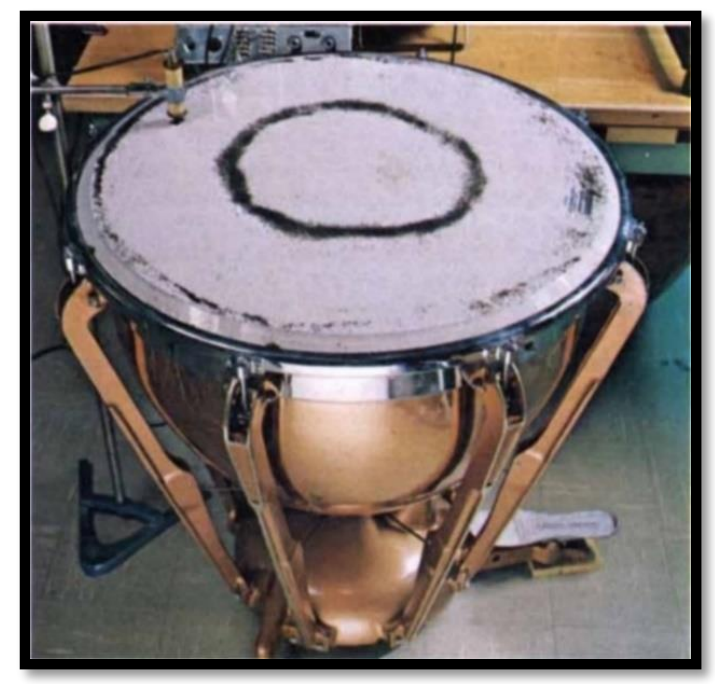

Fig. 3. Standing wave pattern on a kettledrum head. One of many possible standing wave patterns on a kettledrum head, made visible by dark powder sprinkled on the drumhead. As the head is set into oscillation at a single frequency by a mechanical oscillator at the upper left of the photograph, the powder collects at the nodes. By the same way, due to the oscillation of the solar system wave function the dust grains and gas of the solar system protoplanetary disk were grouped in certain orbits.

\footnotetext{
${ }^{7}$ We know from classical mechanics that the elliptic orbits of the planets (Kepler's first law) are the result the Newton's law of gravitation, which is an inverse_square relation.

${ }^{8}$ The wave function of the solar system probably is formed either when the sun was a protostar or when the newborn sun was on the Main-sequence. The distance between these two phases is very short (less than 50 million years) [15] and both phases occurred before the formation of the planets. In both states, I have no idea how or why this wave function formed.

${ }^{9}$ Since the mass distribution in the Protoplanetary disk had not been uniform. During formation of the planets, in some nodes of the solar system wave function, less dusts were collected and in some nodes, more dusts were placed next to each other. Therefore, in some nodes a planet had been formed and in others it had not been formed.
} 


\section{The Elimination of Potential from the Schroedinger Equation}

275 Let's revisit the path we have taken. The waves $A e^{i\left(k x-w t+\emptyset_{0}\right)}, A e^{i\left(k x+w t+\emptyset_{0}\right)}, A e^{i\left(k x-w t-\emptyset_{0}\right)}$ and

276

277

278

279

280

281

282

283

284

285

286

287

288

289

290

291

292

293

294

295

296

297

298

299

300

301

302

303

304

305

306

307

308

309

310

311

312

313

314

$A e^{i\left(k x+w t-\emptyset_{0}\right)}$ are the solutions of Schroedinger equation for a free particle. In an unambiguous process using the superposition principle, we proved that the solar system wave function (equation 1) is also the real part of a solution of the Schroedinger equation for a free particle i.e. $i \hbar \frac{\partial}{\partial t} \psi(x, t)=-\frac{\hbar^{2}}{2 m} \frac{\partial^{2} \psi(x, t)}{\partial^{2} x}$. But the sun is under the gravity effect of the center of the galaxy. Therefore, now, everyone is asking themselves how the sun wave function is related to the Schroedinger equation for a free particle. The answer is quite clear. This shows that the associated wave packet of an object (like sun) is not affected by the potential; and the forces of gravity and Coulomb do not affect the associated wave packet of an object and only affect the particle. This example might not be very interesting; But consider a person who has an umbrella over his head and you pull him towards yourself. This work has no effect on the opening and closing of the umbrella (the wave function of the solar system is also an umbrella above the sun). The change in the wavelength of the associated wave packet of an object is probably related to another factor such as the change in the mass of matter or the other factors that we do not yet know. Thus the observations show that the potential has no effect on the associated wave packet of matter and we must eliminate potential from the Schroedinger equation. This is a shocking result, but there is no escaping it.

Here I need to say the last sentence first. Consider Fig. 5. This is the shape of my atomic model, which is based on the diagram of wave function of the solar system (Fig. 2). This model is a unifying model that includes both the Schrodinger wave function and the Bohr atomic orbits. This new atomic model, in addition to justification of main spectrum of the hydrogen, the normal Zeeman effect and anomalous Zeeman effect also predicts the existence of lines of the secondary spectrum of hydrogen that has never been justified before. I think prediction of existence of the secondary spectrum of hydrogen is a complete success for my atomic model. Achieving this new atomic model requires accepting the elimination of potential from the Schrodinger equation and, as we will see in the next section, the invalidity of the uncertainty principle about waves. It is difficult to accept the elimination of potential from Schrodinger equation at first because it means that many parts of quantum books must either be removed or changed. But let us do not forget that our goal in quantum is description of the nature and behavior of atoms and subatomic particles. In this article, we will show that the description of the nature and behavior of atoms and subatomic particles is possible without the potential in the Schrodinger equation.

In addition, remember that a wrong theory (like Schrodinger equation along with potential) can also justify phenomena. For example, some astronomical observations can be justified by the Ptolemy epicycle model [15], or some phenomena, such as the neutrality of the atom, can be explained by the Thomson atomic model. These were two examples of a wrong theory that could justify phenomena. The Stark effect and the Zeeman effect are justified using the existence of potential in the Schrodinger equation. But for example in justifying of normal Zeeman effect, based on wave mechanics, we do not know what happen in the atom when we apply the magnetic field [19]. But we can use my atomic model to provide a physical picture from what happens when we apply a magnetic field in normal Zeeman effect. 


\section{Uncertainty Principle and Single-Frequency Wave Packet}

316 We obtained the equation of a single-frequency wave packet in the previous sections, both empirically and mathematically (equation 1). But, a thought experiment [20], which is based on Heisenberg uncertainty principle [21], arrives us to this conclusion that a single-frequency wave packet cannot be existed. This thought experiment investigates the beat between two singlefrequency waves. In this thought experiment, it is proved that only a wave with infinite spreading can be single-frequency. This means that a wave packet, which is localized, cannot be single frequency. This thought experiment is apparently unambiguous, but there is a very important point about thought experiments. A thought experiment, even if based on a logical process, may lead to wrong results. We prove this with an example. A well-known example is the famous experiment of Michelson and Morley about the velocity of light [22]. If you do the Michelson and Morley experiment in your mind, you will conclude that the velocity of light depends on the velocity of observer and source. This was something that Michelson and Morley also expected, because according to the logic that governs our human brains, the velocity of light must be combined with the velocity of observer and source. But when experiments were performed in the real world, they concluded that the velocity of light was independent of the motion of observer and source. This example shows that we cannot comment on the results of an experiment until it is not performed in the real world. This example fully proves that a thought experiment is meaningless because you cannot rely on its results and its results may be wrong. Obviously, this rule also is true for the thought experiment mentioned at the beginning of this section. And therefore we cannot comment on the results of this experiment until it is performed. Which it means that we cannot comment on the existence or nonexistence of single-frequency wave packet based on the thought experiment of beat. In addition, attention to the empirical equation 1 we have a single-frequency wave packet and therefore the uncertainty principle is wrong about waves.

339

340

341

342

343

Furthermore, this thought experiment (beat experiment) arrived physicists to spreading of the wave packet that is completely incorrect and wrong. Physicists, based on the mentioned thought experiment, assume that they do not know the form of equation $w$ in terms of $k$, and to make a wave packet, they expand $w$ about $k_{0}$ and write:

$$
w(k)=w\left(k_{0}\right)+\left(k-k_{0}\right)\left(\frac{d w}{d k}\right)_{k_{0}}+\frac{1}{2}\left(k-k_{0}\right)^{2}\left(\frac{d^{2} w}{d k^{2}}\right)_{k_{0}}
$$

Replacing this expression in equation 2 we will obtain an equation which means that a wave packet spreads over time [23][9][24]. The spreading of the matter wave packet is a subject that physicists are unable to justify and it is in stark contrast to the reality of the universe. The spreading of wave function of a particle means that the wave function of the particle must have disappeared long ago, and we should not observe the wave function of any particle today because the particles of the universe like electrons were not created today or this year. Most of them are as old as the universe. Thus, their wave functions had to be so wide in the short time after creation that did not differ from zero and we must be unable to detect theirs effect in the experiment. As a result of it, we should not observe the wave property of the electron in the Davisson-Germer or the Thomson experiments today. The justification for the spreading of wave packet based on probabilities $\left(|\psi(x, t)|^{2}\right)$ is also not acceptable and is an attempt due to helplessness. So we see that the result of the mentioned 
thought experiment (experiment of the beat of two waves) is contrary to reality and the associated wave of the particles should not spread.

The solar system wave function (equation 1) is a non-spreading wave packet. We proved that without the solar system wave packet, it is not possible to reach to the Titius-Bode rule from the molecular nebula and the protoplanetary disk. Furthermore, I will present a new atomic model, which seems to be the final model of the atom (Fig. 5). In this model, electrons rotate on the nodes of the associated wave packet of nucleus, which is a non-spreading wave packet. If we consider equation 16; We will never reach to the non-spreading wave packet of the nucleus and the nonspreading wave packet of the solar system mathematically. Therefore, these two facts also show that the wave packet does not spread.

Aside from this fact that the spreading of the wave packet is unacceptable, as I said, the most important thing is that the results of a thought experiment are not reliable.

\section{9-1. The new Atomic model}

In this section, we present a new atomic model based on the model of the solar system wave function (Fig. 2). This model has both the Schrodinger wave function and the Bohr atomic model inside. Our atomic model explains why the Bohr atomic orbits are quantized. Niels Bohr could not explain this issue. Based on this model, the main spectrum of the hydrogen atom, the secondary spectrum, the normal Zeeman effect, and the other phenomena will be explained in a completely understandable way. Some of the atomic models are shown in Fig. 4.
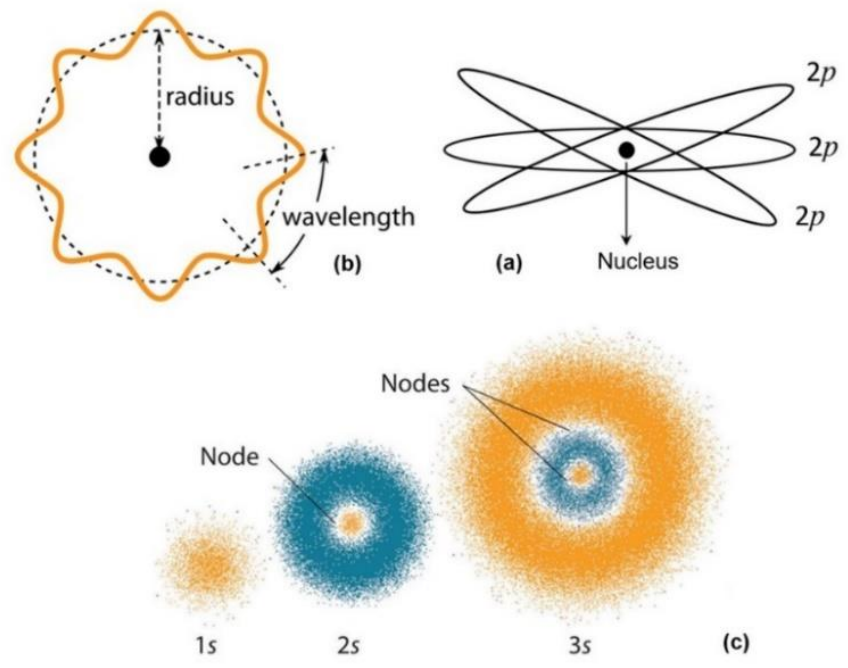

Fig. 4. Some of the atomic model. This figure shows three atomic models: a). Bohr-Sommerfeld atomic model: In this model, as you can see, subshells have space orientation. In this model, the horizontal $2 P$ subshell is in the same plane as the $1 S$ and $2 S$ subshells. $1 S$ and $2 S$ subshells are not shown in this figure. b). De Broglie atomic model and c). the Schrodinger atomic model. In this figure Schrodinger's $S$ orbitals are shown. According to Max Born probability theory, the electron can be found in the distance between the nodes in the Fig. 4c. Other atomic models, such as the Rutherford or Thomson atomic models, are not shown in this figure. 
And figure. 5 shows my atomic model, which I call the "New Atomic Model". To arrive to figure. 5, we used the same method as at the beginning of this article. The Bohr atomic model is a successful model which its orbits are at $x_{1}=0.53 \AA, x_{2}=4 x_{1}, x_{3}=9 x_{1}$, etc. In order to be able to assign a standing wave to Bohr model, our wavelength must be equal to $2 x_{1}=1.06 \AA$. Suppose the first orbit of the new atomic model is the first orbit in the Bohr atomic model, namely $x_{1}=0.53 \AA$. The first orbit in our model is equivalent to the first node and the first node means $\emptyset=\frac{\pi}{2}$ because $\operatorname{Cos} \frac{\pi}{2}=0$. In such a case we have:

$$
x_{1}=0.53 \AA \Rightarrow \psi\left(x_{1}\right)=0 \Rightarrow \cos \left(k x_{1}+\emptyset_{0}\right)=0 \Rightarrow k x_{1}+\emptyset_{0}=\frac{\pi}{2} \stackrel{k=\frac{2 \pi}{1.06}}{\Longrightarrow} \emptyset_{0}=-\frac{\pi}{2}
$$

In the same way as the reasonings at the beginning of the paper, the final form of "hydrogen atom wave function" is equal to:

$$
\begin{cases}\operatorname{Re} \psi(x, t)=2\left(\frac{1}{2 \pi \alpha}\right)^{1 / 4} \cos \left(w_{0}^{\prime} t\right) \cos \left(5.92 x-\frac{\pi}{2}\right) e^{-\frac{x^{2}}{4 \alpha}} & x \geq 0 \\ \operatorname{Re} \psi(x, t)=2\left(\frac{1}{2 \pi \alpha}\right)^{1 / 4} \cos \left(w_{0}^{\prime} t\right) \cos \left(5.92 x+\frac{\pi}{2}\right) e^{-\frac{x^{2}}{4 \alpha}} & x \leq 0\end{cases}
$$

In this equation, we consider the angular frequency equal to $w_{0}^{\prime}$ that not to be confused with $w_{0}$ in equation 14. Figure 5 is drawn in cylindrical coordinate by using equation 17.

The first node of the associated wave packet of the hydrogen nucleus is at $r_{1}=0.53 \AA$ and the second node is at $r_{2}=2 r_{1}$, and Third at $r_{3}=3 r_{1}$, etc. In the new atomic model we have: $r_{n}=n r_{1}$, and in the Bohr model we have $r_{n}=n^{2} r_{1}$. This means that the orbit number $n$ in the Bohr model is the orbit number $n^{2}$ in the new atomic model. For example, the second orbit in the Bohr model is the fourth orbit in the new atomic model (Fig. 6). In figure 5 the electron rotates on the first node of the associated wave packet of the nucleus (just like rotation of planets in Fig. 2). This orbit, like the orbits of the planets in the solar system, is slightly elliptical due to the inverse-square coulomb force of the nucleus. The protuberances in the nucleus wave function are the oscillation of dark matter. As mentioned in the "Elliptical orbits" section, the role of the oscillation of the wave function is to prevent an electron from being placed between the nodes. In the new atomic model, the formation of an atom (for example, a hydrogen atom) is such that the electron approaches the proton and is placed in the first node of the proton associated wave packet. The proton associated wave packet prevents the electron from being placed in the distance between the nodes. Due to the Coulomb force, the electron begins to rotate around the nucleus on an elliptical orbit. In this atomic model we use Bohr's assumptions and assume that the electron does not radiate while it orbits about the nucleus [2]. In this model, like the Bohr model, the spectral lines of the elements are caused by the jump of electrons from the higher orbits to the lower orbits [2], which we will discuss in the next section about it. In fact, the Bohr atomic model is a subset of the new atomic model. 


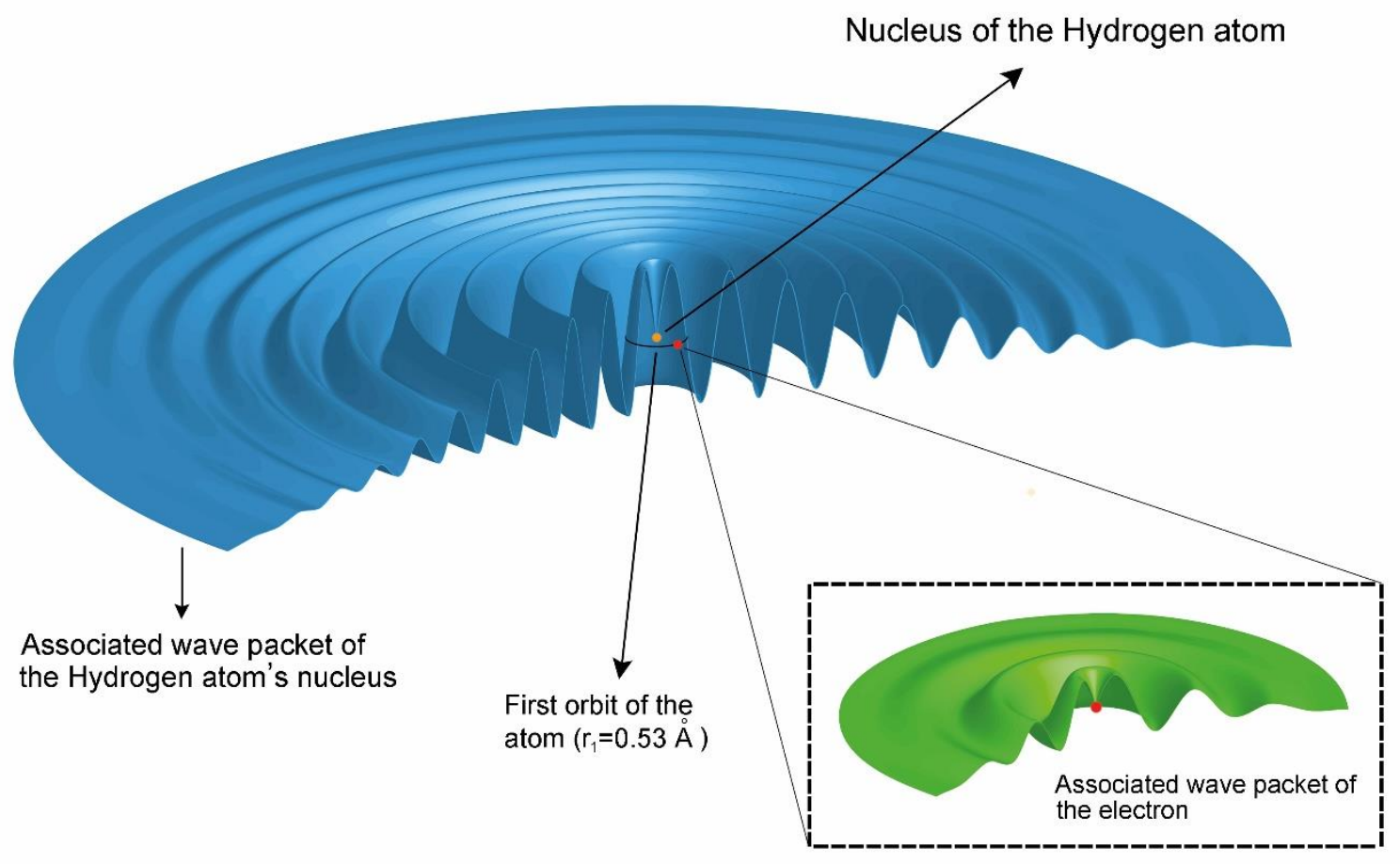

Fig. 5. The New atomic model. In this figure, associated wave packet of nucleus and associated wave packet of the electron are shown at $t=0$. In this figure, the electron and its associated wave rotate about the nucleus of hydrogen atom. The second and the other orbits are not shown in this figure. We consider the wavelength of the nucleus wave packet to be $2 r_{1}\left(r_{1}=0.53 \AA\right)$. If we consider the first orbital velocity of electron based on the Bohr model $v_{1}=\frac{1}{137} c$, in such a case the wavelength of associated wave packet of electron in the first orbit of hydrogen, based on de Broglie equation $\left(\lambda=\frac{h}{m v}\right.$ ), is $3.3 \AA$ (Although, no one has ever measured the velocity of the electron in the first orbit of the hydrogen atom with a laboratory device or method; But $v_{1}=\frac{1}{137} c$ is an empirical value. If $v_{1}$ is not this value, then the precise value of Rydberg constant is not obtained from the Bohr model ). There is no matter if the wavelength of associated wave of electron is larger than or less than the wavelength of the associated wave of the nucleus.

423 In figure 5, The nucleus wave function is plotted using equation 17 with $\alpha=7$ and $\lambda=2 r_{1}=1.06$ $424 \AA$ or $k=5.92$. And the electron wave function with $\lambda=3.3 \AA$ or $k=1.9$ and $\alpha=7$. 


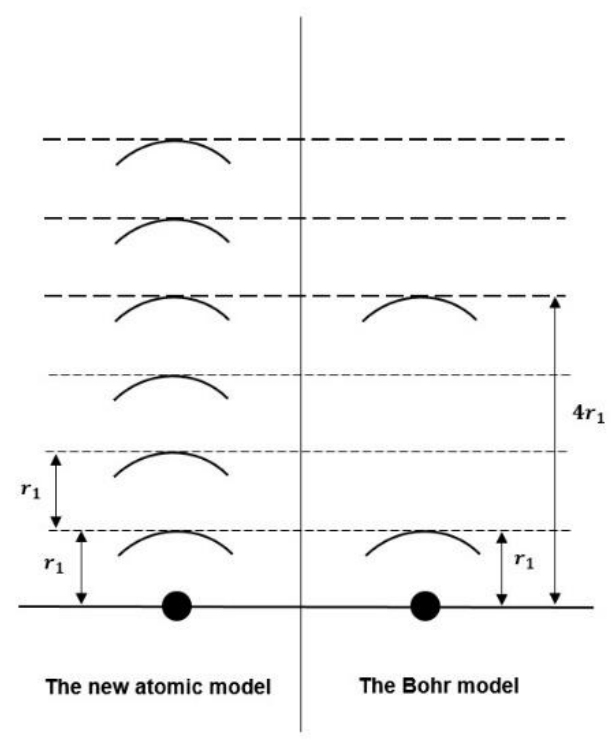

Fig. 6. The new atomic model compared to the Bohr model. For example, the second orbit in the Bohr model is the fourth orbit in the new atomic model.

Regarding Figure 5, we said that the associated wave packet of a particle is dark matter. Dark matter has mass. The mass we measure for elementary particles, such as electron and proton, with different ways is actually the mass of the particle itself and its associated wave packet. Therefore, because the proton is heavier than the electron, its wave packet probably is denser and heavier than the electron wave packet.

In the next section, first we will investigate the motion of electron around the nucleus in a hydrogen atom $\left({ }_{1}^{1} H\right)$ and then, based on the diagram of the first ionization energy of the elements, present our general atomic model and we achieve interesting results.

\section{9-2. The Atomic Hydrogen Model}

We know from mechanics that a mass or an electric charge that is affected by a central force moves on an ellipse which its orbital eccentricity is

Where $E$ is the mechanical energy of the mass $m, L$ is the orbital angular momentum and $K=k_{e} q_{1} q_{2}$ or $K=G M m$ [25]. Semi-major axis of this ellipse is

$$
e=\sqrt{1+\frac{2 E L^{2}}{m K^{2}}}
$$

442 And semi-minor axis is $b=a \sqrt{1-e^{2}}$. The area of this ellipse is $A=\pi a b$ [25].

443 In the motion under the effect of central force $L$ is a constant. And in our new atomic model because 444 of existence of associated wave packet of nucleus, $L$ is also quantized. The amount of $L$ for the 445 first orbit of hydrogen atom is $L_{1 H}=$ Constant. We have: 


$$
L_{1 H}=\text { Constant }, K=k_{e} e^{2} \Rightarrow e_{1 H}=\sqrt{1+\frac{2 E_{1 H} L_{1 H}^{2}}{m_{e}\left(k_{e} e^{2}\right)^{2}}}
$$

$E_{1 H}$ is $13.6 \mathrm{eV}$, based on diagram of first ionization energy. Therefore all of the values in equation 20 are certain values and thus $e_{1 H}$ (namely the orbital eccentricity of first orbit of hydrogen atom) has a certain value. The semi-major axis equals

$$
a_{1 H}=-\frac{K}{2 E_{1 H}} \Rightarrow a_{1 H}=-\frac{k_{e} e^{2}}{2 E_{1 H}}=0.528 \AA
$$

This number is the same radius of the first orbit of the Bohr atom. The semi-minor axis is equal to:

$$
b_{1 H}=a_{1 H} \sqrt{1-e_{1 H}^{2}}
$$

In such a case, the area of orbit equals : $A_{1 H}=\pi b_{1 H} a_{1 H}$. The orbit of electron is a current loop and therefore the orbital magnetic dipole moment for hydrogen atom is equal to:

$$
\boldsymbol{m}_{1 H}=I \boldsymbol{A}_{1 H}
$$

Where $I$ is electric current [26]. In the new atomic model, the direction of the orbital magnetic dipole moment is perpendicular to the plane of the orbit. The dependence of the orbital magnetic dipole moment to the area of the orbit of electron is a very important issue that helps us to justify the normal Zeeman effect. In the following sections, we consider a few subshells for each main orbit, which the areas of these subshells are different from each other. This difference is the cause of difference in the value of $\boldsymbol{m}$, which helps us to justify the normal Zeeman effect. This method is different from Bohr-Somerfield method [27] in old quantum theory. In their model, the normal Zeeman effect is justified by difference in the space orientation of the subshells (Fig. 4a). But in my model, all of the subshells are in the same plane and the difference is in the areas.

\section{9-3. Many-Electron Atoms}

The diagram of the first ionization energy is a very valuable diagram that arrived scientists to many results (Fig. 7). It was by investigation of this diagram that they realized the existence of subshells, the order of subshells filling, and many other results. For example, this diagram shows that in the first shell is not $P$ subshell, or $d$ subshell does not exist in the first and second shells, or in another example, this diagram shows that $4 S$ subshell fills earlier than $3 d$. 


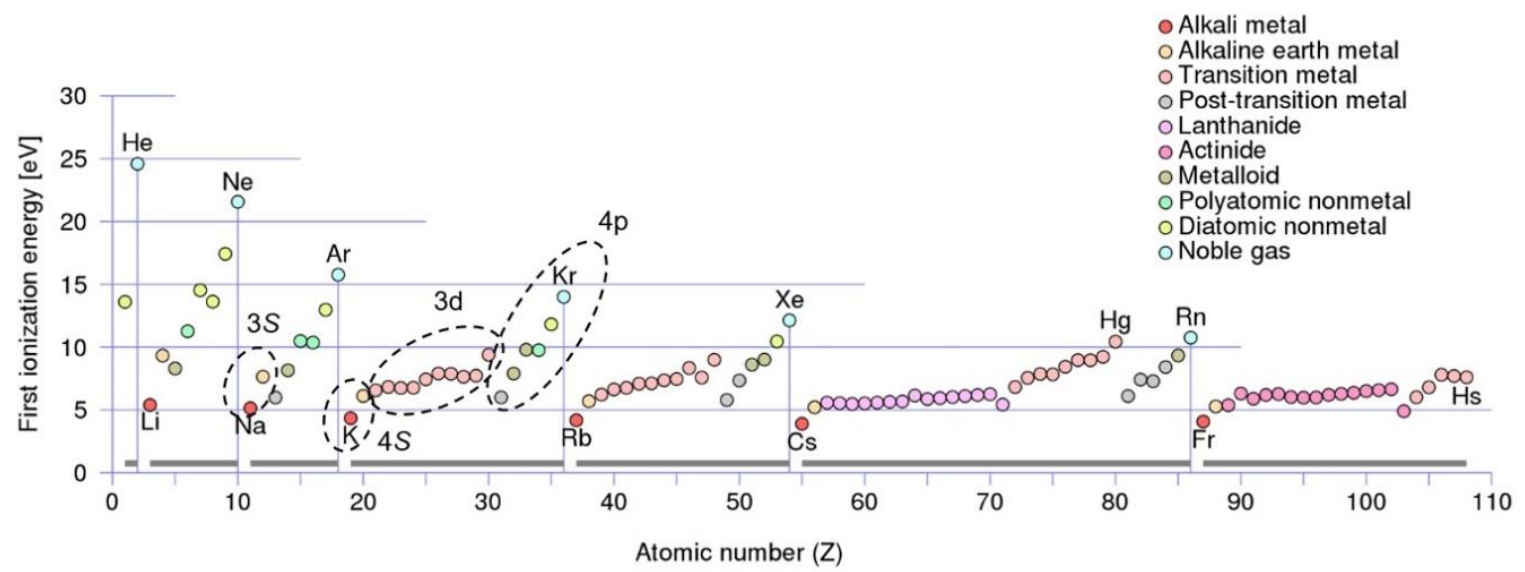

472

473

474

475

476

477

478

479

480

481

482

483

484

485

486

487

488

489

490

491

492

493

494

495

496

497

498

499

500

Fig. 7. The diagram of the first ionization energy.

In the new atomic model, according to the diagram of the first ionization energy and similar to the Bohr-Somerfield model, for each $n$ layer, located at the distance $r_{n}=n r_{1}$; we consider the $S, P$, $d, f$ and $g$ subshells. These are circular and elliptical orbits that have not orientation, unlike the Somerfield model; and all of them are in the same plane and the difference is in their area (the reason for the formation of these subshells can be attributed to the quantized nucleus electric charge and electron charge). The orbital eccentricity and area of these subshells cannot be calculated (except for the hydrogen atom), but we assume that the area of the subshells is not equal. The difference in the area of the subshells cause a difference in the value of the $\boldsymbol{m}$ vector, which causes the occurrence of normal Zeeman effect. For example, the Neon atom has five subshells in the new atomic model. One subshell is $1 S$, another $2 S$ and three $2 P$. All of these subshells are in the same plane, and in each of these subshells, based on the Pauli exclusion principle, only two electrons rotate. Here we consider a numeric index for each of the similar subshells. For example consider $2 P$ subshells. we will have $2 P_{1}, 2 P_{2}$ and $2 P_{3}$.

\section{9-4. Quantum jumps and Spectral Lines of the Atomic Hydrogen}

Before the Bohr atomic model, scientists did not know whether the particles in a hydrogen lamp were molecules or atoms. Because of justification of spectral series such as the Ballmer, Lyman, and Paschen series, by the Bohr atomic model, they understood that most of the particles inside the hydrogen lamp are atoms. But in the hydrogen spectrum, there are other lines besides the main spectrum lines (namely Ballmer, Lyman, .... series.) [3][4][5][6], which are called secondary spectral lines. These lines are not predicted by the Bohr atomic model. By 1922, 750 number of these lines were discovered between $H_{\alpha}$ and $H_{\beta}$ [4] $\left(H_{\alpha}\right.$ and $H_{\beta}$ are the first and second frequencies of the Ballmer series). Some physicists have linked secondary lines to impurities in the hydrogen lamp [3], and some have attributed them to hydrogen molecules in the lamp [3]. But all this was just speculation. My theory considers a large part of these lines to be related to hydrogen atoms and theoretically predicts their existence and gives us their wavelengths. Today, these lines are known as the molecular spectrum of hydrogen [28][29], which this is based on Merton's article [3]. Merton in his article and in the section "Experimental Results" proved in a very vague way 
that two groups of the secondary spectrum lines are related to hydrogen molecules and finally concluded that: "it is probable that the whole of the secondary spectrum is due to the hydrogen molecule". But we show that this is wrong, and only a part of these lines are related to hydrogen molecules.

In the Bohr atomic model, the lines of the emission and absorption spectrum of hydrogen atom are the result of quantum jumps. We use the same assumption in the new atomic theory. Consider Figure 6. As we said, if the Bohr model has $n$ orbits, the new atomic model has $n^{2}$ orbits. For example, the second Bohr orbit is the fourth orbit in the new atomic model (Fig. 6). Based on this, for example, $\mathrm{Ly}_{\alpha}$ in the Lyman series, which equals $v_{21}$ in the Bohr model, is equal to $v_{41}$ in the new atomic model. Since the number of orbits in the new atomic model is more, we should expect more quantum jumps and consequently more emission lines in the hydrogen spectrum. For example, according to Fig. 6, in the new atomic model, we should be able to observe $v_{21}, v_{32}, v_{31}, v_{51}, \ldots$ and many other frequencies, in addition to the lines predicted by the Bohr atomic model. For example, $v_{31}$ in the new atomic model equals

$$
\begin{gathered}
h v_{31}=E_{3}-E_{1} \stackrel{E_{H}=\frac{-\mu k_{e} e^{2}}{2 m_{e} r}}{\Longrightarrow} v_{31}=\frac{1}{1+\frac{m_{e}}{M_{P}}} \frac{k_{e} e^{2}}{2 h}\left(\frac{1}{r_{1}}-\frac{1}{3 r_{1}}\right)=3.35 \times 10^{15} \times \frac{2}{3} \Rightarrow \\
\therefore v_{31}=2.23 \times 10^{15} \mathrm{~Hz} \Rightarrow \lambda_{31}=1340 \AA
\end{gathered}
$$

In the above calculations we used from equation $E_{H}=\frac{\mu}{m_{e}} E_{\infty}=\frac{-\mu k_{e} e^{2}}{2 m_{e} r}=\frac{1}{1+\frac{m_{e}}{M_{P}}} \frac{k_{e} e^{2}}{2 r}$ instead of $E_{\infty}=\frac{-k_{e} e^{2}}{2 r}[30][31]$. The equation $E_{\infty}=\frac{-k_{e} e^{2}}{2 r}$ is the equation of energy of electron when the mass of nucleus is infinite. In the above equation $\mu$ is reduced mass. The Bohr atomic model do not predict the wavelength $\lambda_{31}=1340 \AA$. The observation of this wavelength in the hydrogen spectrum is a strong confirmation for the new atomic theory. As another example, based on the new atomic model, we have:

$$
h v_{114}=E_{11}-E_{4}=\frac{1}{1+\frac{m_{e}}{M_{P}}} \frac{k_{e} e^{2}}{2}\left(\frac{1}{4 r_{1}}-\frac{1}{11 r_{1}}\right) \Rightarrow v_{114}=0.53 \times 10^{15} \mathrm{~Hz} \Rightarrow \lambda_{114}=4640 \AA
$$

This ray is in the visible spectrum region and we should be able to observe it. Another examples are:

$$
h v_{104}=E_{10}-E_{4} \Rightarrow \lambda_{104}=4925 \AA \quad \text { and } \quad \lambda_{84}=5980 \AA
$$

These two wavelengths along with the previous wavelength can be easily seen in Fig. 8. This diagram shows the spectrum of a deuterium arc lamp, instead of a hydrogen lamp. The Deuterium lamp mostly used in spectroscopy, because it provides more intensity at shorter wavelengths and in the ultraviolet region. The wavelengths displayed in the deuterium spectrum are exactly the same as the wavelengths of the hydrogen spectrum with a very slightly shift toward shorter 
wavelengths ${ }^{10}$ [31] (this is due to the very small difference between Rydberg constant for deuterium and hydrogen [31]). In Fig. 8, $D_{\alpha}$ and $D_{\beta}$ are consecutive wavelengths of the Ballmer series. The number of the secondary lines is very large both at short wavelengths and at long wavelengths.

As mentioned, our atomic model (Fig. 5) is based on equation 17. In this equation, the exponential factor is never zero, and therefore the infinite number of orbits can be considered around the nucleus of each atom. By moving away from the nucleus according to equation 17, the effects of the Coulomb force of the nucleus decreases; Therefore, in distant orbits, the electron is practically not bound to the nucleus.

In addition to the new atomic model, some secondary lines are definitely related to hydrogen molecules or impurities inside the lamp.

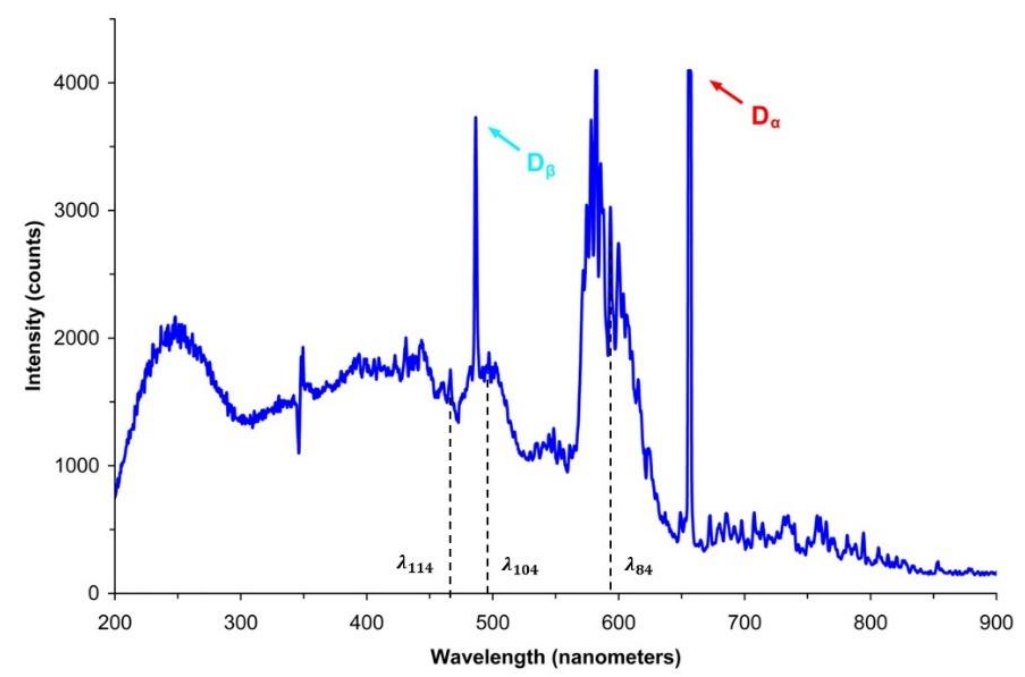

Fig. 8. The spectrum of the deuterium lamp. In this diagram, the position of the wavelengths is shown slightly approximately.

\section{9-5. The Normal Zeeman Effect}

The splitting of spectrum lines in the presence of an external magnetic field, or the normal Zeeman effect (NZE), was discovered by Zeeman in 1896. In the years that followed, Sommerfeld, who justified the fine structure by considering elliptical subshells for each orbit [32], assumed that each of these subshells has a different space orientation and all are not in the one plane [33]. For example, the three $P$ subshells in the second orbit have three orientations (Fig. 4a). As mentioned, in the new atomic model we considered all of the subshells in one plane and assume that these subshells have different areas. Because of difference between these areas the orbital magnetic dipole moments of subshells $(\boldsymbol{m})$ will be different from each other, based on equation 23.

\footnotetext{
${ }^{10}$ This difference is not appearing until three digits after the decimal point
} 
In the following, based on this point, we will explain NZE, in the usual way of proving the NZE in the old quantum mechanics [27]. The difference is that now the cause of the difference in the amount of $\boldsymbol{m}$ is the area of the subshells, not their space orientation.

Consider the third orbit of an atom and its nine subshells and assume that the area of these subshells are as shown in table 2.

\begin{tabular}{|c|c|c|c|c|c|c|c|c|c|}
\hline Subshell of the third orbit & $3 S$ & $3 P_{1}$ & $3 P_{2}$ & $3 P_{3}$ & $3 d_{1}$ & $3 d_{2}$ & $3 d_{3}$ & $3 d_{4}$ & $3 d_{5}$ \\
\hline Area of subshell $\left(A_{\text {subshell }}\right)$ & $20 A_{0}$ & $18 A_{0}$ & $20 A_{0}$ & $22 A_{0}$ & $16 A_{0}$ & $18 A_{0}$ & $20 A_{0}$ & $22 A_{0}$ & $24 A_{0}$ \\
\hline
\end{tabular}

Table. 2. The areas of third orbit's subshells. Here $A_{0}$ is a unit value. In this table the areas of $3 S$, $3 P_{2}$ and $3 P_{3}$ is equal. But there is no problem about this. $3 S$ is a circle; $3 P_{2}$ is an ellipse and $3 P_{3}$ is a more elongated ellipse.

In such a case, the difference in the energy levels in the presence of the magnetic field is obtained from the following equation:

$$
\Delta E_{m}=-\boldsymbol{m}_{\text {subshell }} \cdot \boldsymbol{B}=-I \boldsymbol{A}_{\text {subshell }} \cdot \boldsymbol{B}
$$

Where $\boldsymbol{m}_{\text {subshell }}$ is the orbital magnetic dipole moment of each subshell. If we assume that the direction of $\boldsymbol{B}$ is perpendicular to the orbital plane (or in the direction of $\boldsymbol{A}_{\text {subshell }}$ ), in such a case we have:

$$
\Delta E_{m}=-I A_{\text {subshell }} B
$$

We want to investigate the transition between $d$ and $P$ states in the presence of a magnetic field. When the magnetic field is zero, the energy of the $d$ state is $E_{d}$ (for all five $d$ subshells) and the energy of the $P$ state is $E_{P}$ (for all three P subshells) and because of transition between $d$ and $P$ a photon will be emitted by energy: $h v_{0}=E_{d}-E_{p}$. When the field is turned on, the $d$ state splits into five equally spaced magnetic sublevels, and the $P$ state splits into three equally spaced magnetic sublevels. According to the Table 2 the difference in energy between any two adjacent magnetic sublevels being $\Delta E_{m}=I 2 A_{0} B$. Here we should consider the selection rules. We should assume that the transitions between the subshells follow the rule $\Delta l= \pm 1$; Where the values of $l$ are based on the table below:

\begin{tabular}{|c|c|c|c|c|}
\hline$l=0$ & $l=1$ & $l=2$ & $l=3$ & $l=4$ \\
\hline$S$ & $P$ & $d$ & $f$ & $g$ \\
\hline
\end{tabular}

Table. 3. The subshells and their numbers. Here we use the $l$ sign only because of the historical aspect of the subject, and its connection with Schrodinger mechanics and angular momentum is by no means intended in this section. We 
$\Delta l= \pm 1$ means that the transition from $d$ to $P$ is allowed but from $d$ to $S$ is forbidden. And we also should consider a selection rule for changing area of the subshells. we have:

$$
\Delta A_{l}=0 \text { or } \pm 2 A_{0}
$$

That is, only those transitions are allowed in which either $A_{l}$ does not change or changes by $2 A_{0}$. For changes of $n$ (principal quantum number) any value is allowed. Authorized transitions are shown in Fig. 9.

Magnetic field on

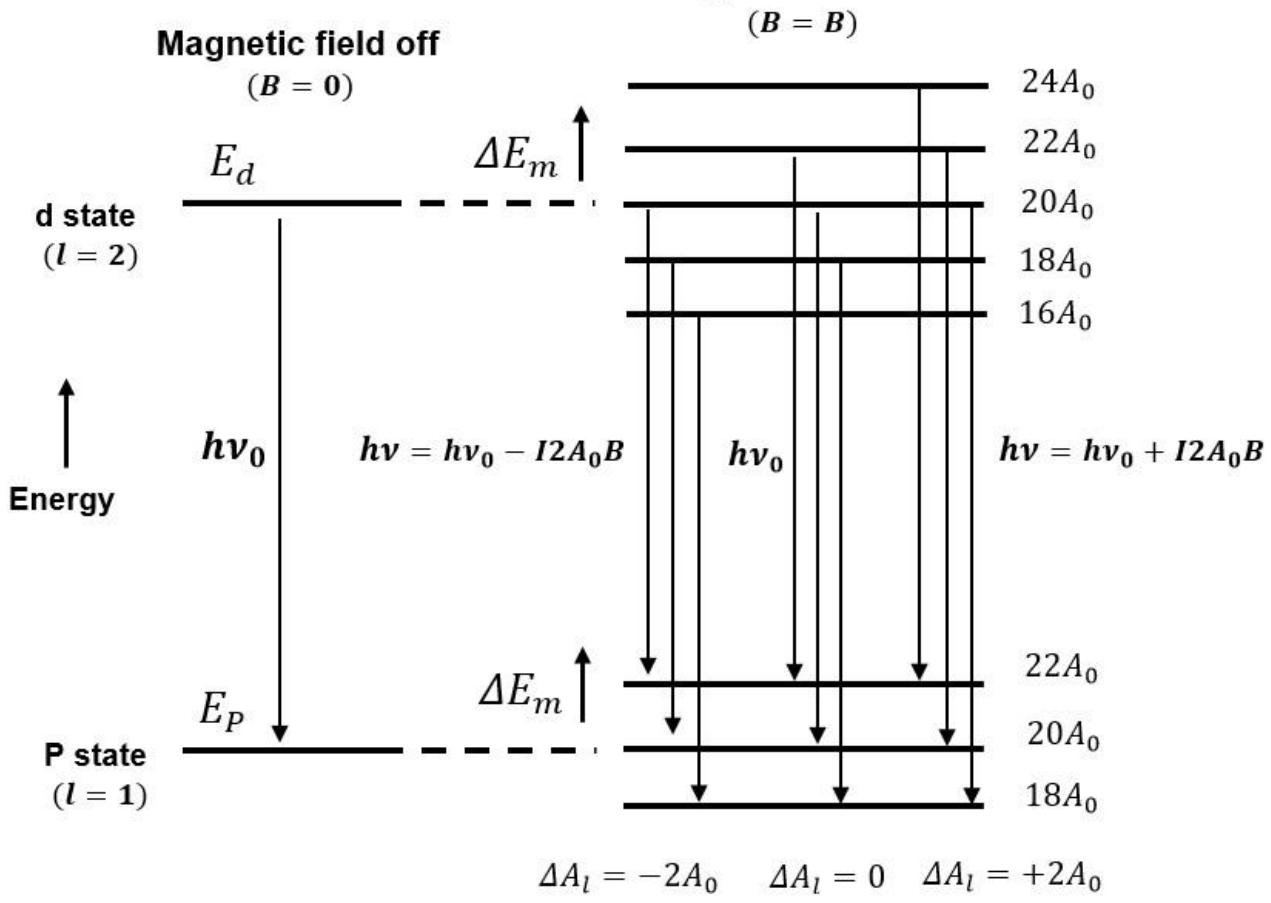

Fig. 9. Schematic illustration of normal Zeeman effect. Energy levels and spectra of $d \rightarrow P$ transition: (left) zero magnetic field. (right) nonzero magnetic field.

We have:

$$
\begin{array}{ll}
\Delta A_{l}=2 A_{0}: & h v=h v_{0}+\Delta\left(\Delta E_{m}\right) \Rightarrow h v=h v_{0}+I 2 A_{0} B \Rightarrow v=v_{0}+\frac{I 2 A_{0} B}{h} \\
\Delta A_{l}=0: & h v=h v_{0}+\Delta\left(\Delta E_{m}\right) \Rightarrow h v=h v_{0} \Rightarrow v=v_{0} \\
\Delta A_{l}=-2 A_{0}: & h v=h v_{0}+\Delta\left(\Delta E_{m}\right) \Rightarrow h v=h v_{0}-I 2 A_{0} B \Rightarrow v=v_{0}-\frac{I 2 A_{0} B}{h}
\end{array}
$$

Thus, each line in the emission spectrum splits into three lines by an external magnetic field. As we said, we used the same common method of proving the NZE [27] here. The difference is that 
here, instead of space orientation of the orbits, the difference in the area of the orbits causes the lines to split. This description of the NZE is easier than the Sommerfeld model.

The above relations were obtained in the situation that $\boldsymbol{B}$ was in the direction of $\boldsymbol{A}$. If $\boldsymbol{B}$ and $\boldsymbol{A}$ have a $\theta$ angle with each other, then in the two equations of above relations we will have $\cos \theta$ :

$$
\begin{array}{ll}
\Delta A_{l}=2 A_{0}: & h v=h v_{0}+I 2 A_{0} B \operatorname{Cos} \theta \Rightarrow v=v_{0}+\frac{I 2 A_{0} B}{h} \operatorname{Cos} \theta \\
\Delta A_{l}=0: & h v=h v_{0} \Rightarrow v=v_{0} \\
\Delta A_{l}=-2 A_{0}: & h v=h v_{0}-I 2 A_{0} B \operatorname{Cos} \theta \Rightarrow v=v_{0}-\frac{I 2 A_{0} B}{h} \operatorname{Cos} \theta
\end{array}
$$

\section{9-6. Spin as a Classical Property}

We know that if we consider the electron as the spinning sphere of charge, in such a case, given the small size of the electron $\left(r<10^{-16} \mathrm{~m}\right)$ [34] and based on the classical equation $L=I \omega, \omega$ will have a large value. A large $w$ means a large peripheral velocity in the equator of the spinning sphere. Which in this case is higher than the velocity of light. But in our model, the electron is surrounded by a wave packet of dark matter (Fig. 5). Dark matter has mass. Thus, This wave has a part of the mass of the electron, and in fact the mass that humans have measured for the electron, by different ways, is the mass of electron and its associated wave. The electron associated wave is much larger than the electron size $\left(r<10^{-16} \mathrm{~m}\right)$. As mentioned, for example, the wavelength of associated wave packet of an electron in the first orbit of a hydrogen atom is 3.3 angstroms. Thus, based on the equation $I=\int r^{2} d m$ the moment of inertia of the electron (namely the moment of inertia of the particle along with its wave packet) increases and according to the equation

$L=\sqrt{s(s+1)} \hbar=\frac{\sqrt{3}}{2} \hbar=I \omega$, $\omega$ decreases (which means that the wave packet of electron, like the electron, has an spining motion). So based on my model, spin can be considered as a classic motion.

Particle experimenters have obtained the diameter of an electron by examining face-to-face collisions [34]. In these experiments, the diameter of the associated wave packet is not measured. The wave packet in collisions is transparent and low effect. The diameter of the wave packet usually is defined as one-half of maximum value of the function at the center.

\section{Conclusion}

In this article we proved that there is almost no doubt about the existence of a wave function in the solar system. Without the solar system wave packet, it is not possible to reach to the TitiusBode rule from the molecular nebula and the protoplanetary disk. De Broglie considered the wave nature of light for subatomic particles, and here we attributed the wave nature to celestial objects. Neither of these two actions is strange. Rather, they are truths that we must become accustomed to. Describing the distances of the planets by a quantum wave packet can be considered as another confirmation of the validity of Quantum theory. 
It seems that the new atomic model is the final model of atom. This model is a unifying model that has both the Schrodinger wave function and the Bohr atomic orbits. In my opinion, prediction of the secondary lines of the hydrogen spectrum is a complete success for the new atomic model. Based on the new atomic model, normal Zeeman effect was more easily justified. Anomalous Zeeman effect, which is caused by the spin of electron, is also easily described by the new atomic model, which I have not described due to the length of the article.

637 Based on this article, the associated wave packet of a matter (such as electron, proton or sun) is one of the candidates for the nature of dark matter.

Here, we proved that the potential must be removed from the Schrodinger equation and we proved that the word of " thought experiment " is meaningless and an experiment must be done in the real world so that we can talk about its results. In this article, we turned quantum mechanics into a simpler and more beautiful theory.

The results and discussions of this article are the beginning of an important change in astronomy, astrophysics, cosmology, quantum mechanics, chemistry and atomic and molecular physics. If we consider the attempts and studies of Einstein, Planck and Bohr as the first step in quantum physics and the works of Heisenberg, Max Born, Schroedinger and Dirac as the second step in quantum physics; it seems that, this article is the third step in the development of quantum physics.

\section{References:}

[1]. Carrol, B. Ostlie, D. An Introduction to Modern Astrophysics (Cambridge University Press, Cambridge, ed. 2, 2017), pp. 716-717

[2]. Bohr, N. On the Constitution of Atoms and Molecules. Philos. Mag. 26, 1, (1913)

[3]. Merton, T. Barratt, S. On the spectrum of hydrogen. Philosophical Transactions of the Royal Society of London.

Series A, Containing Papers of a Mathematical or Physical Character 222: 369-400 (1922)

[4]. Allan, S. The band spectrum of hydrogen. Proc. R. Soc. Lond. 106: 69-82, (1924)

[5]. Nicholson, J. The Secondary Spectrum of Hydrogen. Nature 105, 166-167 (1920)

[6]. Richardson, O. The Secondary Hydrogen Spectrum. Nature 118, 116 (1926)

[7]. Walker, J. Halliday, D. Fundamentals of Physics. (Wiley Ltd, ed. 10, 2007), pp. 449-450

[8]. Branson, Jim. "Fourier Transform of Gaussian", Quantum Physics (April 2003). URL:

https://quantummechanics.ucsd.edu/ph130a/130_notes/node88.html.

[9]. Gasiorowicz, S. Quantum Physics (John Wiley \& Sons Inc, ed. 1, 1974), pp. 27-32

[10]. Cohen-tannoudji, C. Diu, B. Quantum Mechanics. (John Wiley \& Sons Inc, Vol. 1, 1977), pp. 21-22

[11]. Ohanian, H. Modern Physics. (Prentice-Hall, Inc, 1987), pp. 198-200

[12]. Davisson, C. Germer, L. Diffraction of Electrons by a Crystal of Nickel. Phys. Rev. 30, 705. (1927).

[13]. Eisberg, R. Quantum Physics. (John Wiley \& Sons Inc, New York, ed. 2, 1974), pp. 134-135

[14]. Hoffmann, B. The strange story of the Quantum. (Dover Publications, 2011)

[15]. Zeilik, M. Astronomy. Ch. 14 (Cambridge University Press, ed. 9, 2002).

[16]. Bonanno, A. Schlattl, H. Paternò, L. The age of the Sun and the relativistic corrections in the EOS. Astronomy and Astrophysics. 390 (3). 1115-1118 (2002)

[17]. Williams, J. Cieza, L. Protoplanetary Disks and Their Evolution. Annual Review of Astronomy and Astrophysics. 49: 67-117 (2011)

[18]. Rossing, T. The Physics of Kettledrums. Scientific American, 247, No. 5, 172-179 (1982)

[19]. Gasiorowicz, S. Quantum Physics (John Wiley \& Sons Inc, ed. 1, 1974), pp. 209-226

[20]. Weidner, R. Sells, R. Elementary Modern Physics (Allyn and Bacon, ed. 2, 1973), pp 14-16 
674 [21]. Heisenberg, W. Ueber den anschaulichen Inhalt der quantentheoretischen Kinematik and Mechanik. Zeitschrift 675 für Physik 43, 172-198 (1927). English translation in Wheeler and Zurek 1983: 62-84.

676 [22]. Michelson, A. Morley, E. On the Relative Motion of the Earth and the Luminiferous Ether. American Journal 677 of Science. 34 (203): 333-345 (1887)

678 [23]. Darwin, C. Free motion in the wave mechanics, Proc. Roy. Soc (London) A117 (1927).

679 [24]. Cohen-tannoudji, C. Diu, B. Quantum Mechanics. (John Wiley \& Sons Inc, Vol. 1, 1977), pp. 61-66

680 [25]. Arya, A. Introduction to classical mechanics. (Allyn \& Bacon, ed. 1, 1990). pp. 248-251

681 [26]. Ritz, J. Milford, F. Foundations of Electromagnetic Theory. (Addison-Wesley, ed. 3, 1960). pp. 163-165

682 [27]. Weidner, R. Sells, R. Elementary Modern Physics (Allyn and Bacon, ed. 2, 1973), pp 240-247

683 [28]. Dieke, G. The molecular spectrum of hydrogen and its isotopes. Journal of molecular spectroscopy 2, Issues 684 1-6. 494-517 (1958)

685 [29]. Richardson, O. Molecular Hydrogen and its Spectrum. (New Haven, Yale University Press; London, 1934).

686 [30]. Ohanian, H. Modern Physics. (Prentice-Hall, Inc, 1987) pp. 160-161

687 [31]. Eisberg, R. Quantum Physics. (John Wiley \& Sons Inc, New York, ed. 2, 1974), page: 107

688 [32]. Eisberg, R. Quantum Physics. (John Wiley \& Sons Inc, New York, ed. 2, 1974), pp 114-117

689 [33]. Mcevoy, J. Oscar, Z. Introducing Quantum Theory. (Icon Books, ed. 4, 2003)

690 [34]. Eisberg, R. Quantum Physics. (John Wiley \& Sons Inc, New York, ed. 2, 1974), page: 277 